strategies for reducing the risk of these and the risk areas of neonatal nursing practice. It is recommended that risk areas must determine in care of neonatal for reduction of malpractice. The standard of care must describe to promote quality assurance in neonatal setting, special training, skill and knowledge nurses must work in neonatal care.

\section{PARENTS' EXPECTATIONS AND SATISFACTION IN PEDIATRICS WARDS}

doi:10.1136/archdischild-2012-302724.1884

B Eren Fidanci, F Arslan, K Fidanci. Gulhane Military Medical Academy, Ankara, Turkey

Objective The aim of this study is to investigate the expectations of parents about their child's care, the effects of care practices on parents' satisfaction and the relationship between the expectation and the satisfaction.

Design and Methods The research was implemented by using face-to-face interviews with the 256 parents of children at the age of 0-18 years in pediatrics clinic by means of two questionnaires. Parents completed one questionnaire immediately after their child's admission and the second after hospital discharge.

Results As a result mothers are mostly satisfied with the approach of doctors and all their expectations except caring about children's home routines are satisfied by nurses. Supportive approach of health care personal adds a big deal to satisfy parents' expectations.

Conclusion Keeping communication channels open, answering their questions and giving the best care available are respective ways to catch better standards in health care.

\section{FROM FAILURE TO SUCCESS - A CHILD PROTECTION INITIATIVE}

doi:10.1136/archdischild-2012-302724.1885

C Fraser. Paediatric Intensive Care Unit, Great Ormond Street Hospital, London, UK

Background and Aims As for all child-health professionals, knowledge of safeguarding/child protection (SCP) is a key role of PICU nurses. A trust-wide audit showed deficiencies in knowledge of SCP processes in our PICU-lead centre, despite standard training. We describe the institution of a bespoke training package to address this.

Methods After a 2008 audit showed 65\% compliance with SCP documentation, an internal lead nurse for SCP was appointed (CF) and lead a Transformation project entailing a series of bespoke SCP Study Days training ALL staff to Level-3 Child Protection, (recommended NHS London), on-going bi-annual updates, regular SCP information sharing and mandatory SCP induction for new starters.

Repeat audits in 2010 and 2012 are reported, together with further qualitative questions (2012).

Results 30/90 PICU nurses completed each questionnaire. Repeat SCP audit showed increased compliance with SCP processes $65 \%$ (2008), 90\% (2010) and 94\% (2012) with 100\% aware of correct SCP documentation.

All staff now feel supported caring for a child with SCP concerns, and able to suggest areas for improving practice. Consultant Intensivist \& lead social worker were not always identified as people who must be notified.

Conclusions We report increased SCP awareness on our PICU after a lead-nurse transformation project, with nurses now confident managing CP cases, and feeling SCP is well-managed. However, further improvement could be made in nurses interaction with the Hospital Social Care Team and further staff-support when caring for children with SCP concerns would be appreciated - especially when nurses inform parents of SCP referral.

\section{PERCEPTIONS OF FORMALIZED CARE CONFERENCES IN A NICU}

doi:10.1136/archdischild-2012-302724.1886

SN Bojesen. Neonatal Care Unit E 110 N, Herlev Hospital, Herlev, Denmark

Background Implementation of family-centered care (FCC) involves a culture change in relation to nursing structure and method and have great influence on both the physical and psychological environment. Literature shows a connection between physical layout and FCC and a movement of the balance of power and the communication between parents and staff, when the principles of FCC are conducted. The challenges for staff has many facets, ranging from being a guest in the room, to be monitored in action and skills to use appreciative communication. Collaboration and commincation with the parents is decisive.

Aim To explore the perception of formalized care conferences and possible increased parental satifaction and wellbeing.

Methods Structured interviews with mothers, special "Go-karts" and journalaudits on done formalized care confernces.

Results Our survey showed overall a great deal of parental satisfaction but a big differens in the perception of the conferences between staff and mothers. In spite the staff ranked the conferneces "formalized and performed", the mothers didn't experienced the conferences like that - mainly because the majority of the conferences took place nearby the child and randomly. The mothers had a desire for more equal presence to communicate freely.

Conclusions Despite great insight, tools and understanding of the importance of the formalized care conferences, is it difficult for the staff to carry out the conferences as intended. This has revealed the necessity of illuminate the barriers and challenges staff experience in planning and conducting formalized care conferences, which has lead to a new project just started.

\section{WHAT TO DO WHEN THE ALARM BELL RINGS?}

doi:10.1136/archdischild-2012-302724.1887

${ }^{1} \mathrm{M}$ van Leeuwen, ${ }^{1} \mathrm{MCM}$ Vermeulen, ${ }^{2} \mathrm{C}$ van der Starre, ${ }^{1,3} \mathrm{M}$ van Dijk. ${ }^{1}$ Neonatal Intensive Care; 'Intensive Care, Erasmus MC - Sophia Children's Hospital, Rotterdam, The Netherlands; IIntensive Care, Erasmus MC - Sophia Children's Hospital, Rotterdam, Netherlands Antilles

Background and Aim A neonatal intensive care unit (NICU) is a high risk unit for a fire outbreak. To prepare employees for an evacuation they are trained. Here we describe the evacuation drills performed.

Methods Every year a minimum of four evacuation drills are performed at the NICU of the Erasmus MC- Sophia Children's Hospital, Rotterdam. Our NICU consists of 3 separate units. Dolls are used as simulation patients. After each evacuation drill members of the workgroup and participants provide feedback on the effectiveness of the drill.

Results Since 200812 evacuation drills were held for a total of 68 caregivers, (3 to 9 per drill) and 4 workgroup members. The scenarios dealt with fire outbreak $(n=9)$, flooding $(n=2)$ and smoke $(n=1)$. The number of dolls used during evacuation was 1 to 6 . The median time to complete evacuation was 10 minutes (range 7 to $18 \mathrm{~min}$ utes). The outlier of 18 minutes related to a 'patient' whose caseload was high (see Figure). In 9 of the12 drills the alarm bell rang; in two cases nobody in the other units responded. 